\title{
Erratum
}

\section{The Norm Convergence of the Trotter-Kato Product Formula with Error Bound}

\section{Takashi Ichinose ${ }^{1}$, Hideo Tamura ${ }^{2}$}

1 Department of Mathematics, Faculty of Science, Kanazawa University, Kanazawa, 920-1192, Japan. E-mail: ichinose@kenroku.kanazawa-u.ac.jp

2 Department of Mathematics, Faculty of Science, Okayama University, Okayama, 700-8530, Japan. E-mail: tamura@math.okayama-u.ac.jp

Received: 10 June 2003 / Accepted: 19 August 2004

Erratum published online: 2 December 2004 - @ Springer-Verlag 2004

Commun. Math. Phys. 217, 489-502 (2001)

It was kindly pointed out by Vidmantas Bentkus that there is a small gap, in the proof of Lemma 2.1, for the case where $C$ is strictly positive, i.e. $C \geq \eta$ for some constant $\eta>0$.

We have to establish an estimate

$$
\left\|e^{-t S_{\varepsilon}}-e^{-t C}\right\| \leq M t^{-1} \varepsilon^{\alpha}
$$

for every $\varepsilon>0$ with a constant $M$ independent of $t$ and $\varepsilon$. To do so, we need to prove that for $S_{\varepsilon}=\varepsilon^{-1}(1-F(\varepsilon))$, the inverse $S_{\varepsilon}^{-1}$ exists and is uniformly bounded for every $\varepsilon>0$.

The proof given in the paper is correct for sufficiently small $\varepsilon>0$, up to a certain $\varepsilon_{0}>0$, because we can show $S_{\varepsilon}^{-1}$ is uniformly bounded with $\left\|S_{\varepsilon}^{-1}\right\|<2 / \eta$ for all positive $\varepsilon \leq \varepsilon_{0}$. However, we need to supplement that with a proof for the large $\varepsilon$ case. To this end, in the statement of Lemma 2.1, we should have further assumed on $F(t)$, in the case $C$ is strictly positive, that for this $\varepsilon_{0}$ there exists $\delta_{0}=\delta_{0}\left(\varepsilon_{0}\right)>0$ such that $F(t) \leq 1-\delta_{0}\left(\varepsilon_{0}\right)$ for every $t \geq \varepsilon_{0}$, or that for every $\varepsilon>0$ there exists $\delta=\delta(\varepsilon)>0$ such that $F(t) \leq 1-\delta(\varepsilon)$ for every $t \geq \varepsilon$. Hence we can easily see that for these large $\varepsilon, S_{\varepsilon}$ is uniformly bounded with $\left\|S_{\varepsilon}^{-1}\right\|<\varepsilon_{0} / \delta_{0}$.

Such an additional assumption on $F(t)$ in Lemma 2.1, for the case $C$ is strictly positive, does not affect the rest of the proof of the main Theorem. 